\title{
Rapid Screening of Bovine Milk Oligosaccharides in a Whey Permeate Product and Domestic Animal Milks by Accurate Mass Database and Tandem Mass Spectral Library
}

\author{
Hyeyoung Lee ${ }^{\dagger, \perp}$, Daniel J. Cuthbertson ${ }^{\ddagger}$, Don E. Otter§, ${ }^{\S}$, and Daniela Barile ${ }^{\star}, \dagger, \|$ \\ tDepartment of Food Science and Technology, University of California-Davis, Davis, California \\ 95616, United States ${ }^{\ddagger}$ Agilent Technologies, Inc., Santa Clara, California 95051, United States \\ $\S$ School of Chemical Sciences, University of Auckland, Auckland 1142, New Zealand "Foods for \\ Health Institute, University of California-Davis, Davis, California 95616, United States
}

\begin{abstract}
A bovine milk oligosaccharide (BMO) library, prepared from cow colostrum, with 34 structures was generated and used to rapidly screen oligosaccharides in domestic animal milks and a whey permeate powder. The novel library was entered into a custom Personal Compound Database and Library (PCDL) and included accurate mass, retention time, and tandem mass spectra. Oligosaccharides in minute-sized samples were separated using nanoliquid chromatography (nanoLC) coupled to a high resolution and sensitive quadrupole-Time of Flight (Q-ToF) MS system. Using the PCDL, 18 oligosaccharides were found in a BMO-enriched product obtained from whey permeate processing. The usefulness of the analytical system and BMO library was further validated using milks from domestic sheep and buffaloes. Through BMO PCDL searching, 15 and 13 oligosaccharides in the BMO library were assigned in sheep and buffalo milks, respectively, thus demonstrating significant overlap between oligosaccharides in bovine (cow and buffalo) and ovine (sheep) milks. This method was shown to be an efficient, reliable, and rapid tool to identify oligosaccharide structures using automated spectral matching.
\end{abstract}

\section{Keywords}

bovine milk oligosaccharides; tandem mass spectral library; whey permeate; cow milk; sheep milk; buffalo milk

\footnotetext{
*Corresponding Author: Tel: +1-530-752-0976. Fax: +1-530-752-4759. dbarile@ucdavis.edu.

$\perp$ Present Address: H.L.: Division of Metrology for Quality of Life, Korea Research Institute of Standards and Science, Yuseong, Daejeon 34113, Republic of Korea.

\#Present Address:D.E.O.: Center for Dairy Research, University of Wisconsin-Madison, Wisconsin 53706, United States.

Supporting Information

The Supporting Information is available free of charge on the ACS Publications website at DOI: 10.1021/acs.jafc.6b02039.

Tables of sheep and buffalo milk oligosaccharides (PDF)

Notes

The authors declare no competing financial interest.
} 


\section{INTRODUCTION}

Milk oligosaccharides are a class of nondigestible carbohydrates consisting of at least three monosaccharides linked by various glycosidic bonds. The compositions of human and bovine milk oligosaccharides have been extensively studied because of a widespread interest in their bioactive properties. Recent studies reported their biological efficacy as prebiotics and anti-infectives. ${ }^{1,2}$ The biosynthesis of oligosaccharides in mammalian cells follows complex pathways where monosaccharide units are sequentially added by the activity of specific glycosyltransferases. They are structurally complicated molecules made of either lactose or lactosamine cores decorated with galactose, $\mathrm{N}$-acetylhexosamine, sialic acid, and fucose. ${ }^{3,4}$ Because monosaccharides are attached to the core via different glycosidic bonds, oligosaccharides are diverse in terms of chemical structure. Theoretically, because oligosaccharides can form glycosidic linkages at any hydroxyl site on a given monosaccharide, an incredible diversity of oligosaccharides are formed, based on the combinations of possible monosaccharides. This diversity arises from complex, nontemplate-based biosynthesis, involving several enzymes. ${ }^{5}$ The length and branching of an oligosaccharide depends on the monosaccharides and enzymes available at the time of their assembly. In certain biological samples, such as bovine milk, a limited number of oligosaccharides are produced, due to limited glycosyl-transferase expression.

In glycomics, novel resources and technologies have been developed to investigate glycan structure-function relationships. ${ }^{6-9}$ Among them, mass spectrometry (MS) has become the method of choice because of its sensitivity and speed of characterization of oligosaccharide structures. ${ }^{10,11}$ Additionally, high-performance liquid chromatography (HPLC) is a technique used to separate oligosaccharides in a complex mixture based on their retentive properties. ${ }^{12}$ A combination of HPLC and MS technologies provides accurate mass, isotope pattern, and retention time information. However, a more-detailed linkage analysis of each oligosaccharide is necessary for rigorous assignment of the structures. One approach involves the use of exoglycosidase enzymes that specifically cleave individual monosaccharides from the nonreducing end of the oligosaccharide. The shifts in the chromatographic retention time upon treatment with each glycosidase are used to assign the structure based on the specificity of cleavage. ${ }^{13-15}$ However, this approach is rather expensive (requiring the use of all the possible hydrolases) and extremely time-consuming, requiring a prefractionation of the sample and multiple MS analyses for each fraction after the sequential enzymatic treatments. A more rapid throughput approach involves the use of tandem mass spectrometry (MS/MS), which enables assignment of fine structures with fragmentation pattern arising from a unique mass signature. ${ }^{16,17}$ Recent studies show that some bovine milk oligosaccharide (BMO) structures in colostrums and mature milks were elucidated with the combination of the techniques mentioned above. ${ }^{15,18,19}$ However, the techniques for the fine structure characterization of oligosaccharides have limitations when considering the highly heterogeneous oligosaccharide samples. The requirement of large sample amounts, multiple enzymatic steps, and MS/MS fragmentation methods complicates the use of these techniques for high-throughput analysis. There is still a need for a large amount of manual data analysis, which requires extensive knowledge of carbohydrate chemistry and mass spectrometry. To facilitate rapid and improved identification of an 
ensemble of oligosaccharide structures, an alternative approach uses a reference library for oligosaccharides in certain biological matrices together with their analytical characteristics (e.g., accurate mass, isotope pattern, retention time, and MS/MS spectra). Recently, a human milk oligosaccharide (HMO) library was constructed and used to rapidly identify unknown oligosaccharides by comparing the fragmentation spectra. ${ }^{20}$

The present study details the use of a nanoliquid chromatographic (nanoLC) separation coupled to a high resolution and sensitive quadrupole-Time of Flight (Q-ToF) MS system for the detection of over 30 BMO that were previously elucidated. ${ }^{15}$ Each BMO with known formulas was individually characterized to determine retention time, observed spectral molecular weight, and characteristic fragmentation pattern. The data generated were used to build a custom database and library that were used to screen unknown samples. The library was applied to a commercial whey permeate product and different milks from domestic animals.

\section{MATERIALS AND METHODS}

\section{Materials and Chemicals}

Bovine colostrum samples were collected from Jersey and Holstein cows within $12 \mathrm{~h}$ of calving. The samples were pooled and frozen at $-80^{\circ} \mathrm{C}$. A BMO-enriched bovine whey permeate powder was supplied by Hilmar Ingredients (Hilmar, CA). Sheep and buffalo milk samples were provided by AgResearch (New Zealand). The milks were collected from the animals at the end of their first month of lactation. Porous graphite carbon cartridges were purchased from Alltech Associated (Deerfield, IL). Sodium borohydride and trifluoroacetic acid were purchased from Sigma-Aldrich (St. Louis, MO). All other reagents used were either of analytical or HPLC grade.

\section{Sample Preparation of Oligosaccharides from Bovine Colostrum, a Whey Permeate Product, and Sheep/Buffalo Milks}

Identification of free oligosaccharides by MS requires their separation from the other components in the mixture. The oligosaccharides in the samples were extracted as described previously, with slight modifications. ${ }^{15,21}$ A $500-\mu \mathrm{L}$ aliquot of sample was centrifuged for $30 \mathrm{~min}$ at $15,000 \mathrm{~g}$ and $4^{\circ} \mathrm{C}$. The top fat layer was removed, leaving the oligosaccharide-rich bottom layer. The powdered whey permeate sample was dissolved in water $1 \%(\mathrm{w} / \mathrm{v})$. Water $(500 \mu \mathrm{L})$ and $4 \mathrm{~mL}$ of Folch solution (chloroform/methanol 2:1, v/v) were added to the defatted samples. The mixture was centrifuged for $30 \mathrm{~min}$ at $4,000 \mathrm{~g}$ and $4^{\circ} \mathrm{C}$. The aqueous top layer containing oligosaccharides was collected, and ethanol was added at a 2:1 ratio. The mixture was kept at $4^{\circ} \mathrm{C}$ overnight to precipitate the remaining protein and then centrifuged. The oligosaccharide-rich fraction was dried in a vacuum centrifuge (miVac, Genevac, Ipswich, UK). The dried oligosaccharides were resolubilized in $2 \mathrm{~mL}$ of $1.0 \mathrm{M}$ sodium borohydride and kept at $60^{\circ} \mathrm{C}$ for $1 \mathrm{~h}$ to chemically reduce the oligosaccharides from aldehydes to alditols. The samples were further purified using graphitized carbon cartridges ( $4 \mathrm{~mL}$, Alltech, Deerfield, IL). Prior to use, the cartridges were conditioned with three column volumes of $80 \%$ acetonitrile in $0.05 \%$ aqueous trifluoroacetic acid and another three column volumes of deionized water. The oligosaccharide-containing samples were loaded 
onto the column and washed with five column volumes of deionized water. The oligosaccharides retained by the solid-phase extraction were eluted with five column volumes of $40 \%$ acetonitrile in $0.1 \%$ aqueous trifluoroacetic acid. The samples were dried in vacuo.

\section{nanoHPLC-Chip/Q-ToF MS Analysis}

Oligosaccharides were analyzed using an Agilent 6520 HPLC-Chip/quadrupole Time-ofFlight (Chip/Q-ToF) MS system (Agilent Technologies, Inc., Santa Clara, CA) equipped with a microwell-plate autosampler (maintained at $4^{\circ} \mathrm{C}$ ), capillary sample loading pump, nano pump, HPLC-Chip/MS interface, and the ToF MS detector. The chip used consisted of a $9 \times 0.075 \mathrm{~mm}$ i.d. enrichment column and a $43 \times 0.075 \mathrm{~mm}$ i.d. analytical column, both packed with $5 \mu \mathrm{m}$ porous graphitized carbon as the stationary phase. Chromatographic separation was performed by a binary gradient consisting of mobile phase A, 3\% acetonitrile in water (v/v) with $0.1 \%$ formic acid, and mobile phase B, $90 \%$ acetonitrile in water $(\mathrm{v} / \mathrm{v})$ with $0.1 \%$ formic acid. The gradient conditions were as previously reported. ${ }^{15}$ The dried samples were dissolved in $1 \mathrm{~mL}$ of water and diluted 10-fold for sheep milk and 100-fold for bovine colostrum and the whey permeate product, and $1 \mu \mathrm{L}$ aliquots were injected. The mass acquisition ranges were $m / z 450-2500$ in MS mode and $m / z 50-1500$ in MS/MS mode. The instrument was operated in $2 \mathrm{GHz}$, extended dynamic range with a dual nebulizer ESI source. Sample data were collected in the positive ion mode using the HPLC-Chip interface. Mass accuracies are typically $<5 \mathrm{ppm}$ for MS and $<20 \mathrm{ppm}$ for MS/MS analyses. To ensure adequate resolution and mass accuracy, reference mass 922.0098 was monitored.

The collision energy applied was based on the following equation:

$$
\text { Collision energy }=\frac{m \times z}{100} \times 1.3-3.5
$$

\section{Data Analysis}

Oligosaccharide identification was performed using Agilent MassHunter Qualitative Analysis software (version B.06.00). Individual oligosaccharides were identified by two approaches, and the results were compared. First, only accurate mass and retention time were used for the assignment of oligosaccharide. Second, an accurate mass database with a spectral library in personal compound database and library format (PCDL) (Agilent Technologies, Inc., Santa Clara, CA) was compared with experimentally obtained data. Oligosaccharide structures were identified based on accurate masses, retention times, and MS/MS spectra.

In the first approach, MS scans provided accurate masses, which were used to assign composition, using a search algorithm, such as the glycan finder program (in-house). Briefly, as the experimental masses were inserted, the search algorithm examined a list of experimentally measured masses and searched for all possible monosaccharide combinations matching the experimental mass within $<10 \mathrm{ppm}$ mass errors. Relative quantitation was performed by measuring the peak area of the corresponding extracted ion chromatogram. 
For the second method, BMO were identified by screening the accurate mass compound database and MS/MS spectral library. We built the PCDL format database and spectral library to contain BMO names, molecular formula, retention times, structures, and MS/MS spectra. Using a "Find by Molecular Features" algorithm, the Qualitative Analysis software extracted all of the possible compounds from each analysis based on $\mathrm{m} / z$ and retention time. Then, the database and library were applied, and they reported those BMOs whose retention time difference and mass errors were within restrictive set criteria (mass error $<10 \mathrm{ppm}$; retention time window $<2 \mathrm{~min}$ ). The experimental MS/MS spectra were compared with the spectral data in the library. A match score was calculated for MS/MS spectra, as well as mass error, isotope spacing, and isotope abundances. More information is presented in a previous publication..$^{20}$

\section{RESULTS AND DISCUSSION}

\section{Building a BMO Personal Compound Database and Library (PCDL)}

A database of tandem MS composed of 34 BMO structures was constructed (Table 1). The structures included in this study were characterized and elucidated in an earlier study by our group. ${ }^{15}$ Among $50 \mathrm{BMO}$ reported in the literature, the detailed structures, including linkage, monosaccharide type, and composition, were identified in 34 BMO structures. We also referred to other publications by Urashima et al. (21 BMO), Marino et al. (37 BMO), and Albrecht et al. (33 BMO). ${ }^{19,22-24}$ Some structures reported were aided by nuclear magnetic resonance. ${ }^{22,25,26}$ The tandem MS database and library are composed of accurate mass, chromatographic retention time, and tandem mass spectra of each BMO, along with the collision energy applied. All criteria correspond to unique characteristics. Regarding the precursor ion identification, due to the high mass resolution of Time-of-Flight (ToF) instruments, accurate mass provides the complete monosaccharide composition. In positive ionization mode, most BMOs are observed as the protonated molecular ions, $[\mathrm{M}+\mathrm{H}]^{+}$or $[\mathrm{M}+2 \mathrm{H}]^{2+}$, and the list of BMO accompanied by neutral masses is detailed in the database. A graphitized carbon LC stationary phase was employed for the baseline HPLC separation for isomers. ${ }^{27,28}$ A representative chromatogram of bovine colostrum BMO used in this study is presented in Figure 1A. Acidic oligosaccharides, including sialyllactose (SL) and sialyllactosamine (SLN), are predominant, and also a large portion of neutral tri- and tetrasaccharides is observed. This is consistent with previous studies. ${ }^{18,29} \mathrm{It}$ is worth noting that the chromatographic column enabled sufficient separation of sets of isomeric BMO, including 6 $6^{\prime} \mathrm{SL}$ (retention time $=13.7 \mathrm{~min}$ ) and $3^{\prime} \mathrm{SL}$ (retention time $=20.2 \mathrm{~min}$ ). The separations of other milk oligosaccharides were demonstrated in previous publications. 15,20,28 An additional level of discrimination can be achieved through the fragmentation of the parent ions. A detailed list of the proposed product ions along with the relative abundance is listed in the library (Table 1). BMOs underwent fragmentation resulting in common fragment ions of monosaccharides such as hexose $(\mathrm{m} / z$ 183.09), hexosamine $(\mathrm{m} / z$ 204.09), and $\mathrm{N}$-acetylneuraminic acid (Neu5Ac, $\mathrm{m} / z$ 292.10). Additional glycosidic and internal cleavages were generated, providing information related to oligosaccharide sequence and branching, therefore generating characteristic tandem MS spectra for each oligosaccharide. The ability to distinguish structures by tandem MS is demonstrated with two sialyllactose (SL) isomers: 3'SL and 6'SL (Figure 2). Both SL spectra showed $\mathrm{B}_{1}, \mathrm{~B}_{2}$, 
$\mathrm{Y}_{1}$, and $\mathrm{Y}_{2}$ fragment ions; however, their relative intensities differed. $\mathrm{B}$ type ions were major fragment ions in $3^{\prime} \mathrm{SL}$, whereas $\mathrm{Y}_{1}$ ion was the most intense in $6^{\prime} \mathrm{SL}$ spectra. The intensities of the fragment ions in tandem MS experiments are the result of a complex combination of factors such as the relative stability of precursors and products, bond strengths, activation barriers, or position of the charge. ${ }^{30}$ All these factors are in turn the consequence of a specific chemical structure. Hence, even when isomers can yield the same products in tandem MS experiments, they can still be resolved based on the relative abundances of characteristic fragment ions. ${ }^{31}$ To determine whether the corresponding structures could be identified using this method in a straightforward fashion, the MS/MS spectra in the library were compared with those found in bovine milk via the library search. In both comparisons, the correct isomer yielded the highest scores; the scores for $6^{\prime} \mathrm{SL}$ and $3^{\prime} \mathrm{SL}$ were $94 \%$ and $98 \%$, respectively (Table $2 \mathrm{a}, \mathrm{b}$ ).

\section{Application of the BMO Database and Spectral Library to a Whey Permeate Product}

Whey permeate is a coproduct of cheese-making obtained after the removal of protein from whey. When cheese whey is passed through an ultrafiltration membrane to concentrate protein, smaller molecules cross through the membrane to yield the whey permeate. Recently, we demonstrated that whey permeate is a good source of prebiotic milk oligosaccharides. ${ }^{21,32,33}$ The massive industrial quantities of bovine dairy streams can make large-scale isolation of oligosaccharides possible. Therefore, whey permeate is considered to be a source of functional compounds for novel product development. The database and library were tested and validated using an industrial BMO product from whey permeate. Several well-known BMO were observed in the product; however, the relative intensities of the major BMO appeared different from those in bovine colostrum (Figure 1). This can be explained by the fact that mature milk, not colostrum, is used to produce cheese, and it is well-known that oligosaccharide composition evolves during lactation. ${ }^{29,34,35}$ Tentative assignment of oligosaccharides (with monosaccharide composition) showed that, in the whey permeate product, there were 38 potential oligosaccharide compositions including isomers (Table 3a). For comparative purpose, the tandem MS library was adopted as an additional identification tool. An extracted ion chromatogram for each compound in the database (within a specified mass window, e.g., $538.206 \pm 10 \mathrm{ppm}$ for $[\mathrm{LNnH}+2 \mathrm{H}]^{2+}$ ) was used to integrate the chromatogram to position compounds and to compare the isotope ratio of the mass spectra for each peak with the theoretical isotope ratio (Figure 3A-C). A further level of analyte discrimination can be achieved through the fragmentation of the compound position in the sample chromatogram, comparing the resultant product spectra with that stored in the spectral library (Figure 3D-E). A score was assigned from each of the search criteria, and the combination of the scores produced a match score. For example, the database score was $96 \%$, and the library score was $85 \%$. Therefore, the combined match score for $\mathrm{LNnH}$ (lacto- $N$-neohexaose) in the whey permeate product was $91 \%$. The PCDL searching results produced $18 \mathrm{BMO}$ that were assigned with their structures to their match scores (Table 3b).

Barile et al. ${ }^{20}$ reported 15 oligosaccharide compositions in whey permeate in 2009, and in a following publication, Dallas et al. ${ }^{21,32}$ assigned 24 oligosaccahrides, not including isomers. To the best of our knowledge, the present study is the first to show the many isomeric forms 
of BMO in whey permeate with their structural details. $3^{\prime} \mathrm{SL}$ was the most abundant oligosaccharide in bovine whey permeate, followed by $3^{\prime}$-sialyl-galactosyl-lactose, $6^{\prime} \mathrm{SL}(\mathrm{Neu} 5 \mathrm{Ac}), 6^{\prime} \mathrm{SLN}(\mathrm{Neu} 5 \mathrm{Ac}$ ), triose B, $N$-acetylglucosaminyl-lactose, and sialyllacto- $N$ tetraose (LST) in order of descending abundance (Table $3 \mathrm{~b}$ ). By employing the programassisted spectral matching, the entire identification process can be done quickly with high confidence.

\section{Application of BMO Database and Spectral Library to Domestic Animal Milks}

Our custom database and library were further validated using milks from two additional domestic animals: sheep and buffalo. These species were chosen because they are ruminants, herbivores, and have digestive systems similar to those of cows. These milk samples were prepared and analyzed in the same way as the bovine milk samples were. Because of the low oligosaccharide concentration in the sheep and buffalo milk samples, the analyses were carried out using limited cleanup steps to limit oligosaccharides losses, which led to a more complex chromatogram that included non-oligosaccharide peaks (Figure 4). ${ }^{36}$ A MS scan was conducted to obtain accurate masses that were used to assign oligosaccharide composition. Seventeen oligosaccharide compositions were identified in sheep milk samples (Supplemental Table 1a). Ovine milk oligosaccharides were composed of short oligomeric chains. Sialic acid residues included both Neu5Ac and $\mathrm{N}$-glycolylneuraminic acid (Neu5Gc), and interestingly, the latter was significantly more abundant with the proportion of 24/76 (Neu5Ac/Neu5Gc), whereas bovine milk contained more Neu5Ac than Neu5Gc. ${ }^{18}$ An automated tandem MS library search comparing the MS/MS spectra from the ovine milk sample was conducted. The library search produced $15 \mathrm{BMO}$ with match scores above $70 \%$ (Supplemental Table 1b). The prominent peaks at 10.4 and 10.8 min corresponded to the triose $\mathrm{B}(\mathrm{Gal}(\beta 1-3) \mathrm{Gal}(\beta 1-4) \mathrm{Glc})$ and triose $\mathrm{C}(\mathrm{Gal}(\beta 1-6) \mathrm{Gal}(\beta 1-4) \mathrm{Glc})$ with the scores $95 \%$ and $97 \%$, respectively (neutral mass: 506.18). The structures and relative abundances of the two isomers were consistent with reports in earlier papers that trioses were the most abundant neutral oligosaccharides in sheep milk and that $\mathrm{Gal}(\beta 1-3) \mathrm{Gal}(\beta 1-4) \mathrm{Glc}$ and $\operatorname{Gal}(\beta 1-6) \mathrm{Gal}(\beta 1-4) \mathrm{Glc}$ constituted the predominant isomeric form. ${ }^{24,37}$ We also confirmed the presence of a fucosylated oligosaccharide $\left(2^{\prime} \mathrm{FL}\right)$ with a confident match score of $83 \%{ }^{24}$ In addition, triose $\mathrm{A}$, triose $\mathrm{D}, \mathrm{LNnT}, \mathrm{LNnH}$, and $\mathrm{LNnP1}$ were found as minor neutral oligosaccharides. ${ }^{24}$ In regard to anionic oligosaccharides, five Neu5Gc-containing oligosaccharides were found in MS only mode (Supplemental Table 1a); nonetheless, two of them, $3^{\prime} \mathrm{SL}\left(\mathrm{Neu} 5 \mathrm{Gc}\right.$ ) and $6^{\prime} \mathrm{SLN}(\mathrm{Neu} 5 \mathrm{Gc}$ ), were assigned by a library search (Supplemental Table 1b). ${ }^{24,38}$ Another three Neu5Gc-containing oligosaccharides may have unique structures that were not detected in bovine milk; therefore, the structures were not searchable using the BMO library. ${ }^{38}$ Four Neu5Ac-containing oligosaccharides were found and assigned: 6'SL(Neu5Ac), 3'SL(Neu5Ac), 6'SLN(Neu5Ac), and 3' -sialyl-galactosyllactose with the match scores of $93 \%, 94 \%, 77 \%$, and $70 \%$, respectively. They were also reported to be present in sheep milk. ${ }^{24}$

A buffalo milk sample was also analyzed in the same way as the ovine milk sample. Accurate mass analysis revealed 18 oligosaccharide compositions (Supplemental Table 2a). Neither fucosylated nor Neu5Gc-containing oligosaccharides were found in the buffalo milk. All anionic oligosaccharides contained only Neu5Ac. This finding could be explained by the 
lack of the activity of CMP- $N$-acetylneuraminic acid hydroxylase in buffalo mammary glands. ${ }^{39}$ In Supplemental Table 2b, 13 oligosaccharides were assigned with detailed structures using the database and spectral library search. To the best of our knowledge, this is the first report of a buffalo milk oligosaccharide profile. $6^{\prime} \mathrm{SL}(\mathrm{Neu} 5 \mathrm{Ac})$ was the major anionic oligosaccharide in buffalo milk with a match score of 94\%, and 3'SL(Neu5Ac), $6^{\prime} \mathrm{SLN}(\mathrm{Neu}$ Ac), and 3' -sialyl-galactosyl-lactose were also present. Regarding neutral oligosaccharides, four trisaccharides (triose B, triose $\mathrm{C}$, triose $\mathrm{D}$, and $N$-acetylglucosaminyllactose) were found with their structural details. In addition, more complex neutral oligosaccharides such as LNnH, 40210, LNnP1, 20110, and LNnT were identified with confident match scores of $70 \%, 71 \%, 95 \%, 74 \%$, and $72 \%$, respectively. The composition of $3 \mathrm{Hex}+2 \mathrm{HexNAc}$ was determined based on mass accuracies of less than $10 \mathrm{ppm}$ in MS scan mode; however, it was not assigned by the library search, which means that the structure was not present in cow's milk. The structure can be $\operatorname{GlcNAc}(\beta 1-3) \operatorname{Gal}(\beta 1-4) \operatorname{GlcNAc}-(\beta 1-$ $3) \mathrm{Gal}(\beta 1-4) \mathrm{Glc}$, the connectivity of which was elucidated in a previous publication. ${ }^{40}$

In this study, the accurate mass database and tandem mass spectra library were generated and used for the identification of BMO in several milk samples. The combination of highly reproducible LC, high mass accuracy MS, and high quality tandem MS allowed confident compound identification. The library contains $34 \mathrm{BMO}$ with details of mass and retention time values. The identities of the oligosaccharides were further confirmed by accurate mass MS/MS analysis on the Q-ToF MS. This program assisted structural identification in a rapid and simple way by matching to a MS/MS spectral library. Comparing the results from accurate mass only versus the spectral library search, we found that the automatic spectral library search provided accurate assignment of oligosaccharide structures without false positive results. A great degree of the homology of milk oligosaccharides was found among the cow, ovine, and buffalo milks.

Given the expandable nature of the database, additional compounds can be included in the database by the simple addition of a molecular formula and MS/MS spectra. This would enable newly acquired sample chromatograms to be interrogated in the event of milk oligosaccharide metabolites, which are not currently part of the database, being identified using a complementary technique. Therefore, future application would comprise analyzing body fluids derived from clinical studies. A growing number of clinical studies are being carried out with BMO supplements: the novel tool described here would be useful for tracking the metabolic fate of oligosaccharides in blood, urine, and stool, and would be of pivotal importance to validate the observed activities. ${ }^{41,42}$ The ability to automate the database makes it an attractive tool for milk oligosaccharide research as it is not possible to predict the biological activity of oligosaccharides a priori without knowing their structural characteristics.

\section{Supplementary Material}

Refer to Web version on PubMed Central for supplementary material.

\section{Acknowledgments}

We thank Hilmar Ingredients for donating the whey permeate product and C. J. Dillard for editing this manuscript. 
Funding

This research was supported in part by funding from the Bill and Melinda Gates Foundation, National Institutes of Health awards R21AT006180, R01AT007079, R01AT008759, USDA:-NIFA 2014-05266, and the Peter J. Shields Endowed Chair in Dairy Food Science.

\section{References}

1. Zivkovic AM, Barile D. Bovine milk as a source of functional oligosaccharides for improving human health. Adv Nutr. 2011; 2:284-289. [PubMed: 22332060]

2. Barile D, Rastall RA. Human milk and related oligosaccharides as prebiotics. Curr Opin Biotechnol. 2013; 24:214-219. [PubMed: 23434179]

3. Mehra R, Kelly P. Milk oligosaccharides: structural and technological aspects. Int Dairy J. 2006; 16:1334-1340.

4. Boehm G, Stahl B. Oligosaccharides from Milk. J Nutr. 2007; 137:847S-849S. [PubMed: 17311985]

5. Lowe JB, Marth JD. A genetic approach to mammalian glycan function. Annu Rev Biochem. 2003; 72:643-691. [PubMed: 12676797]

6. Raman R, Raguram S, Venkataraman G, Paulson JC, Sasisekharan R. Glycomics: an integrated systems approach to structure-function relationships of glycans. Nat Methods. 2005; 2:817-824. [PubMed: 16278650]

7. Hashimoto K, Goto S, Kawano S, Aoki-Kinoshita KF, Ueda N, Hamajima M, Kawasaki T, Kanehisa M. KEGG as a glycome informatics resource. Glycobiology. 2006; 16:63R-70R.

8. Lütteke T, Bohne-Lang A, Loss A, Goetz T, Frank M, von der Lieth C-W. GLYCOSCIENCES.de: an Internet portal to support glycomics and glycobiology research. Glycobiology. 2006; 16:71R$81 \mathrm{R}$.

9. von der Lieth C-W, Freire AA, Blank D, Campbell MP, Ceroni A, Damerell DR, Dell A, Dwek RA, Ernst B, Fogh R, Frank M, Geyer H, Geyer R, Harrison MJ, Henrick K, Herget S, Hull WE, Ionides J, Joshi HJ, Kamerling JP, Leeflang BR, Lütteke T, Lundborg M, Maass K, Merry A, Ranzinger R, Rosen J, Royle L, Rudd PM, Schloissnig S, Stenutz R, Vranken WF, Widmalm G, Haslam SM. EUROCarbDB: an open-access platform for glycoinformatics. Glycobiology. 2011; 21:493-502. [PubMed: 21106561]

10. Alley WR, Novotny MV. Structural glycomic analyses at high sensitivity: a decade of progress. Annu Rev Anal Chem. 2013; 6:237-265.

11. Kailemia MJ, Ruhaak LR, Lebrilla CB, Amster IJ. Oligosaccharide analysis by mass spectrometry: a review of recent developments. Anal Chem. 2014; 86:196-212. [PubMed: 24313268]

12. Ruhaak LR, Lebrilla CB. Advances in analysis of human milk oligosaccharides. Adv Nutr. 2012; 3:406S-414S. [PubMed: 22585919]

13. Xie Y, Tseng K, Lebrilla C, Hedrick J. Targeted use of exoglycosidase digestion for the structural elucidation of neutral O-linked oligosaccharides. J Am Soc Mass Spectrom. 2001; 12:877-884. [PubMed: 11506219]

14. Wu S, Tao N, German JB, Grimm R, Lebrilla CB. Development of an Annotated Library of Neutral Human Milk Oligosaccharides. J Proteome Res. 2010; 9:4138-4151. [PubMed: 20578730]

15. Aldredge DL, Geronimo MR, Hua S, Nwosu CC, Lebrilla CB, Barile D. Annotation and structural elucidation of bovine milk oligosaccharides and determination of novel fucosylated structures. Glycobiology. 2013; 23:664-676. [PubMed: 23436288]

16. Joshi HJ, Harrison MJ, Schulz BL, Cooper CA, Packer NH, Karlsson NG. Development of a mass fingerprinting tool for automated interpretation of oligosaccharide fragmentation data. Proteomics. 2004; 4:1650-1664. [PubMed: 15174134]

17. Tang H, Mechref Y, Novotny MV. Automated interpretation of MS/MS spectra of oligosaccharides. Bioinformatics. 2005; 21:i431-i439. [PubMed: 15961488]

18. Tao N, DePeters EJ, Freeman S, German JB, Grimm R, Lebrilla CB. Bovine Milk Glycome. J Dairy Sci. 2008; 91:3768-3778. [PubMed: 18832198] 
19. Marino K, Lane JA, Abrahams JL, Struwe WB, Harvey DJ, Marotta M, Hickey RM, Rudd PM. Method for milk oligosaccharide profiling by 2-aminobenzamide labeling and hydrophilic interaction chromatography. Glycobiology. 2011; 21:1317-1330. [PubMed: 21566017]

20. Wu S, Salcedo J, Tang N, Waddell K, Grimm R, German JB, Lebrilla CB. Employment of tandem mass spectrometry for the accurate and specific identification of oligosaccharide structures. Anal Chem. 2012; 84:7456-7462. [PubMed: 22867103]

21. Barile D, Tao N, Lebrilla CB, Coisson J-D, Arlorio M, German JB. Permeate from cheese whey ultrafiltration is a source of milk oligosaccharides. Int Dairy J. 2009; 19:524-530. [PubMed: 20161544]

22. Urashima T, Saito T, Nakamura T, Messer M. Oligosaccharides of milk and colostrum in nonhuman mammals. Glycoconjugate J. 2001; 18:357-371.

23. Urashima T, Taufik E, Fukuda K, Asakuma S. Recent Advances in Studies on Milk Oligosaccharides of Cows and Other Domestic Farm Animals. Biosci, Biotechnol Biochem. 2013; 77:455-466. [PubMed: 23470761]

24. Albrecht S, Lane JA, Marino K, Al Busadah KA, Carrington SD, Hickey RM, Rudd PM. A comparative study of free oligosaccharides in the milk of domestic animals. Br J Nutr. 2014; 111:1313-1328. [PubMed: 24635885]

25. Saito T, Itoh T, Adachi S. Chemical structure of three neutral trisaccharides isolated in free form from bovine colostrum. Carbohydr Res. 1987; 165:43-51. [PubMed: 3664520]

26. Urashima T, Saito T, Ohmisya K, Shimazaki K. Structural determination of three neutral oligosaccharides in bovine (Holstein-Friesian) colostrum, including the novel trisaccharide; GalNAc alpha 1-3Gal beta 1-4Glc. Biochim Biophys Acta, Gen Subj. 1991; 1073:225-229.

27. Brokl M, Hernández-Hernández O, Soria AC, Sanz ML. Evaluation of different operation modes of high performance liquid chromatography for the analysis of complex mixtures of neutral oligosaccharides. J Chromatogr A. 2011; 1218:7697-7703. [PubMed: 21628061]

28. Ruhaak LR, Deelder A, Wuhrer M. Oligosaccharide analysis by graphitized carbon liquid chromatography-mass spectrometry. Anal Bioanal Chem. 2009; 394:163-174. [PubMed: 19247642]

29. Gopal PK, Gill HS. Oligosaccharides and glycoconjugates in bovine milk and colostrum. Br J Nutr. 2000; 84(Suppl 1):69-74.

30. Barton SJ, Whittaker JC. Review of factors that influence the abundance of ions produced in a tandem mass spectrometer and statistical methods for discovering these factors. Mass Spectrom Rev. 2009; 28:177-187. [PubMed: 18680189]

31. Guerrero A, Lebrilla CB. New strategies for resolving oligosaccharide isomers by exploiting mechanistic and thermochemical aspects of fragment ion formation. Int J Mass Spectrom. 2013; 354-355:19-25.

32. Dallas DC, Weinborn V, de Moura Bell JMLN, Wang M, Parker EA, Guerrero A, Hettinga KA, Lebrilla CB, German JB, Barile D. Comprehensive peptidomic and glycomic evaluation reveals that sweet whey permeate from colostrum is a source of milk protein-derived peptides and oligosaccharides. Food Res Int. 2014; 63:203-209. [PubMed: 25284962]

33. Lee H, MeloSilva VLd, Liu Y, Barile D. Quantification of carbohydrates in whey permeate products using high-performance anion-exchange chromatography with pulsed amperometric detection. J Dairy Sci. 2015; 98:7644-7649. [PubMed: 26364096]

34. Martin-Sosa S, Martin MJ, Garcia-Pardo LA, Hueso P. Sialyloligosaccharides in human and bovine milk and in infant formulas: variations with the progression of lactation. J Dairy Sci. 2003; 86:5259. [PubMed: 12613848]

35. Tao N, DePeters EJ, German JB, Grimm R, Lebrilla CB. Variations in bovine milk oligosaccharides during early and middle lactation stages analyzed by high-performance liquid chromatography-chip/mass spectrometry. J Dairy Sci. 2009; 92:2991-3001. [PubMed: 19528576]

36. Martinez-Ferez A, Rudloff S, Guadix A, Henkel CA, Pohlentz G, Boza JJ, Guadix EM, Kunz C. Goats' milk as a natural source of lactose-derived oligosaccharides: Isolation by membrane technology. Int Dairy J. 2006; 16:173-181. 
37. Urashima T, Saito T, Nishimura J, Ariga H. New galactosyllactose containing $a$-glycosidic linkage isolated from ovine (Booroola dorset) colostrum. Biochim Biophys Acta, Gen Subj. 1989; 992:375-378.

38. Nakamura T, Urashima T, Nakagawa M, Saito T. Sialyllactose occurs as free lactones in ovine colostrum. Biochim Biophys Acta, Gen Subj. 1998; 1381:286-292.

39. Ghaderi D, Taylor RE, Padler-Karavani V, Diaz S, Varki A. Implications of the presence of Nglycolylneuraminic acid in recombinant therapeutic glycoproteins. Nat Biotechnol. 2010; 28:863867. [PubMed: 20657583]

40. Saksena R, Deepak D, Khare A, Sahai R, Tripathi LM, Srivastava VM. A novel pentasaccharide from immunostimulant oligosaccharide fraction of buffalo milk. Biochim Biophys Acta, Gen Subj. 1999; 1428:433-445.

41. Meli F, Puccio G, Cajozzo C, Ricottone GL, Pecquet S, Sprenger N, Steenhout P. Growth and safety evaluation of infant formulae containing oligosaccharides derived from bovine milk: a randomized, double-blind, noninferiority trial. BMC Pediatr. 2014; 14:1-11. [PubMed: 24387002]

42. Simeoni U, Berger B, Junick J, Blaut M, Pecquet S, Rezzonico E, Grathwohl D, Sprenger N, Brüssow H, The Study, T. Szajewska H, Bartoli JM, Brevaut-Malaty V, Borszewska-Kornacka M, Feleszko W, François P, Gire C, Leclaire M, Maurin JM, Schmidt S, Skórka A, Squizzaro C, Verdot JJ. Gut microbiota analysis reveals a marked shift to bifidobacteria by a starter infant formula containing a synbiotic of bovine milk-derived oligosaccharides and Bifidobacterium animalis subsp. lactis CNCM I-3446. Environ Microbiol. 2016; 18:2185-2195. [PubMed: 26626365] 
(A)

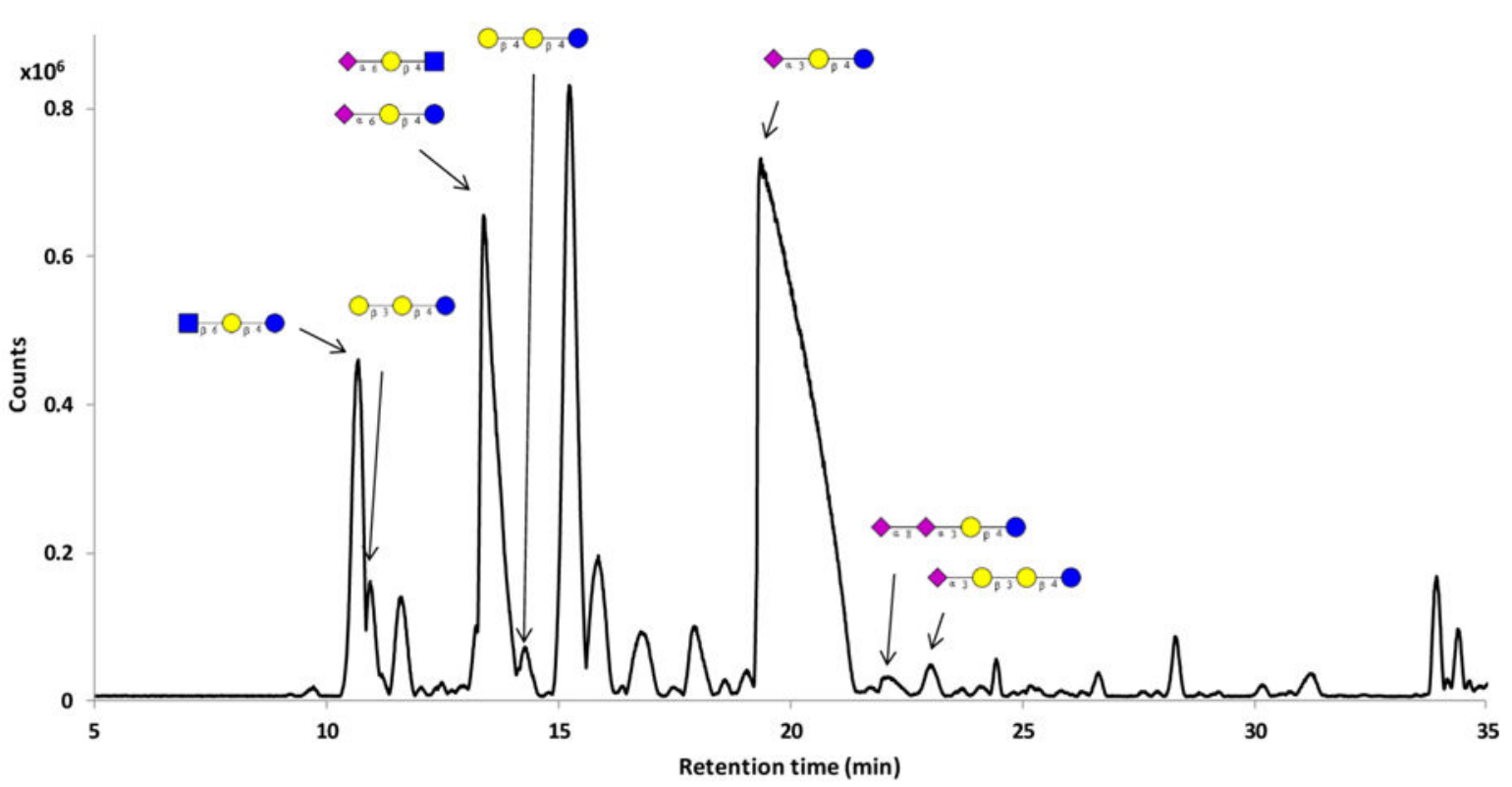

(B)

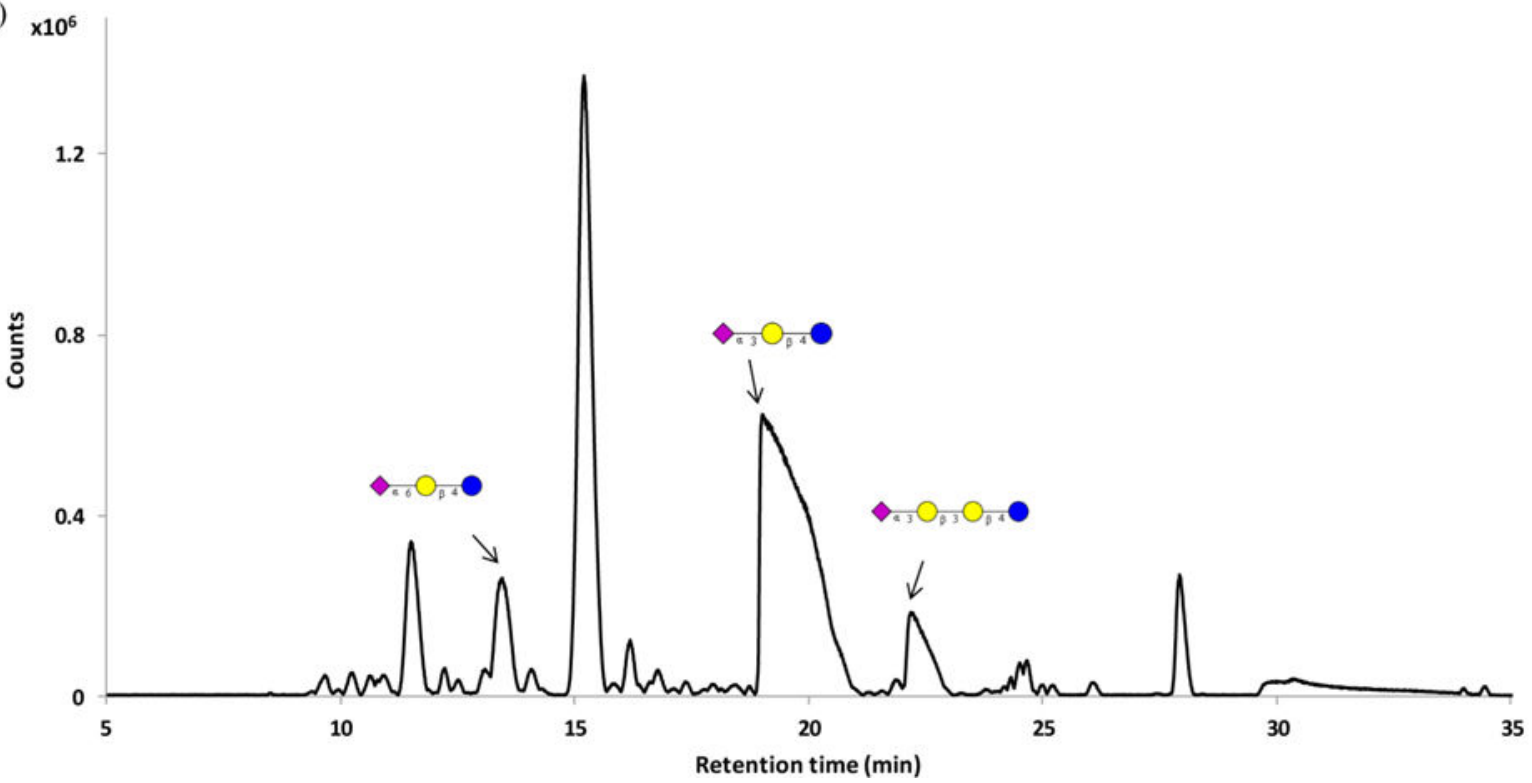

Figure 1.

Representative base peak chromatograms of oligosaccharides from (A) bovine colostrum and (B) a whey permeate product with some structures annotated. 
(A)

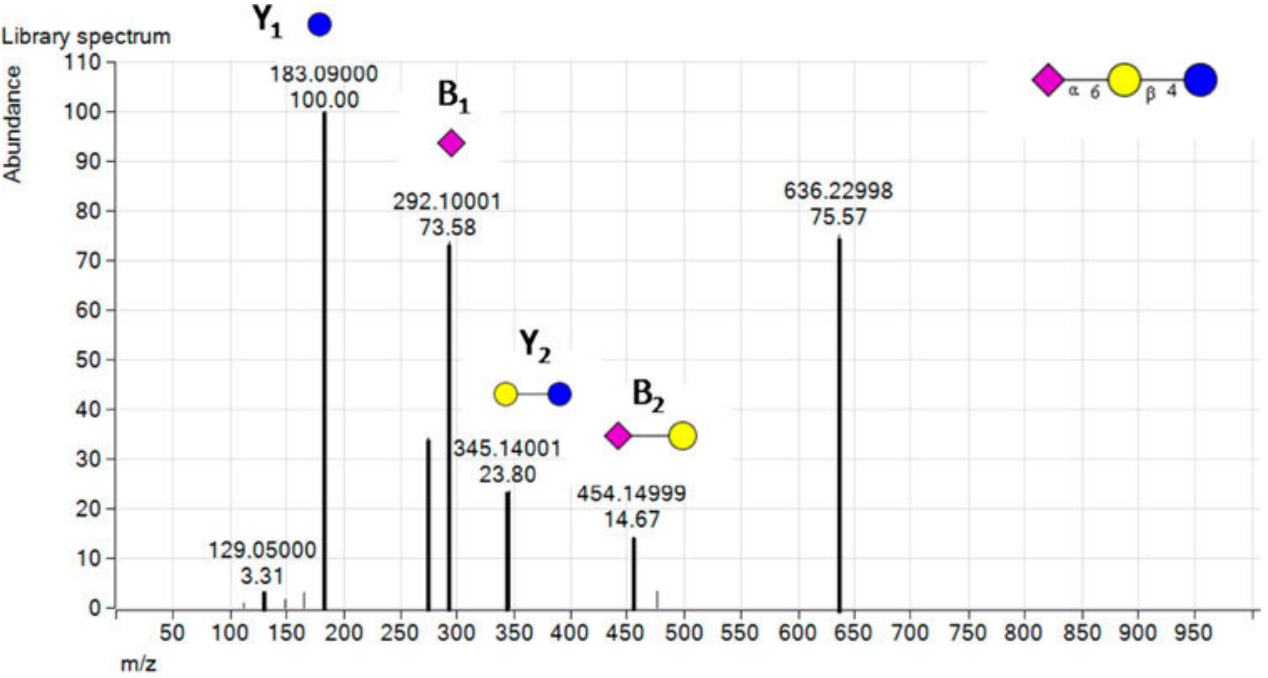

(B)

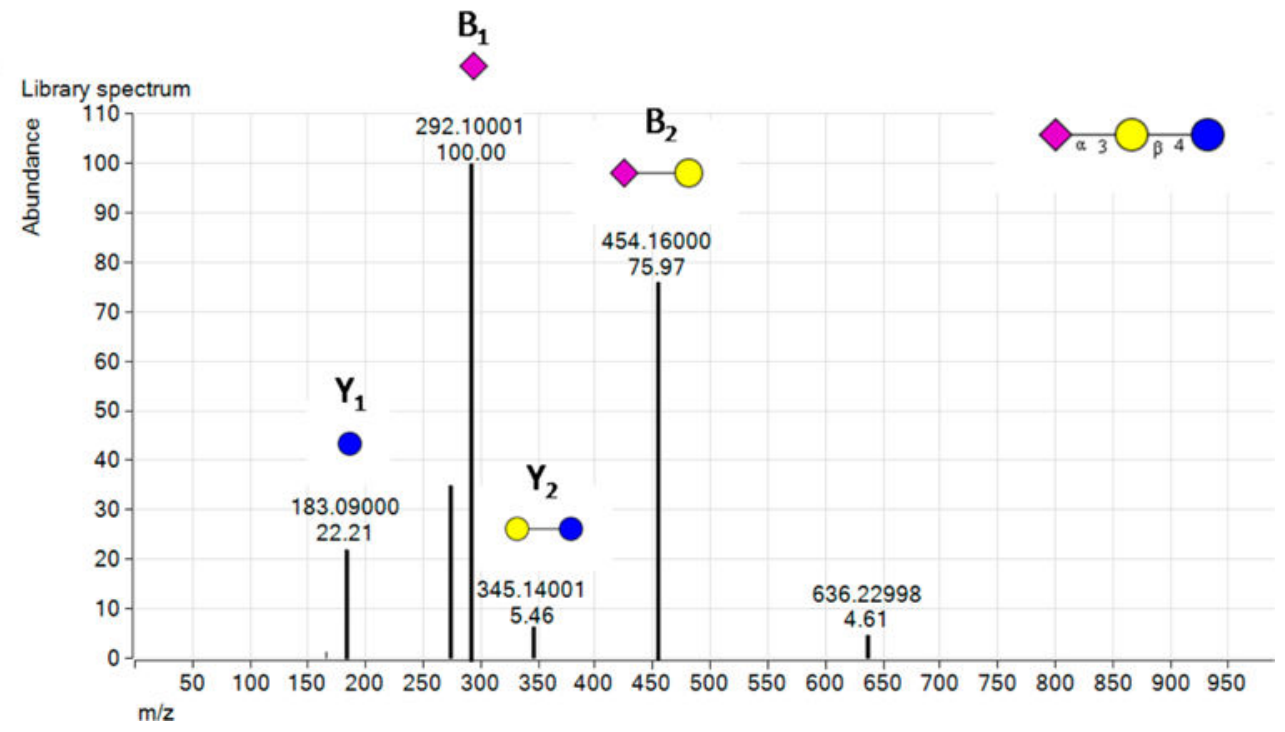

Figure 2.

MS/MS fragmentation patterns for two sialyllactose (SL) isomers: (A) 6'SL and (B) 3' SL with the precursor at $m / z 636.236$. The collision energy used was $4.8 \mathrm{eV}$.

J Agric Food Chem. Author manuscript; available in PMC 2018 March 01. 

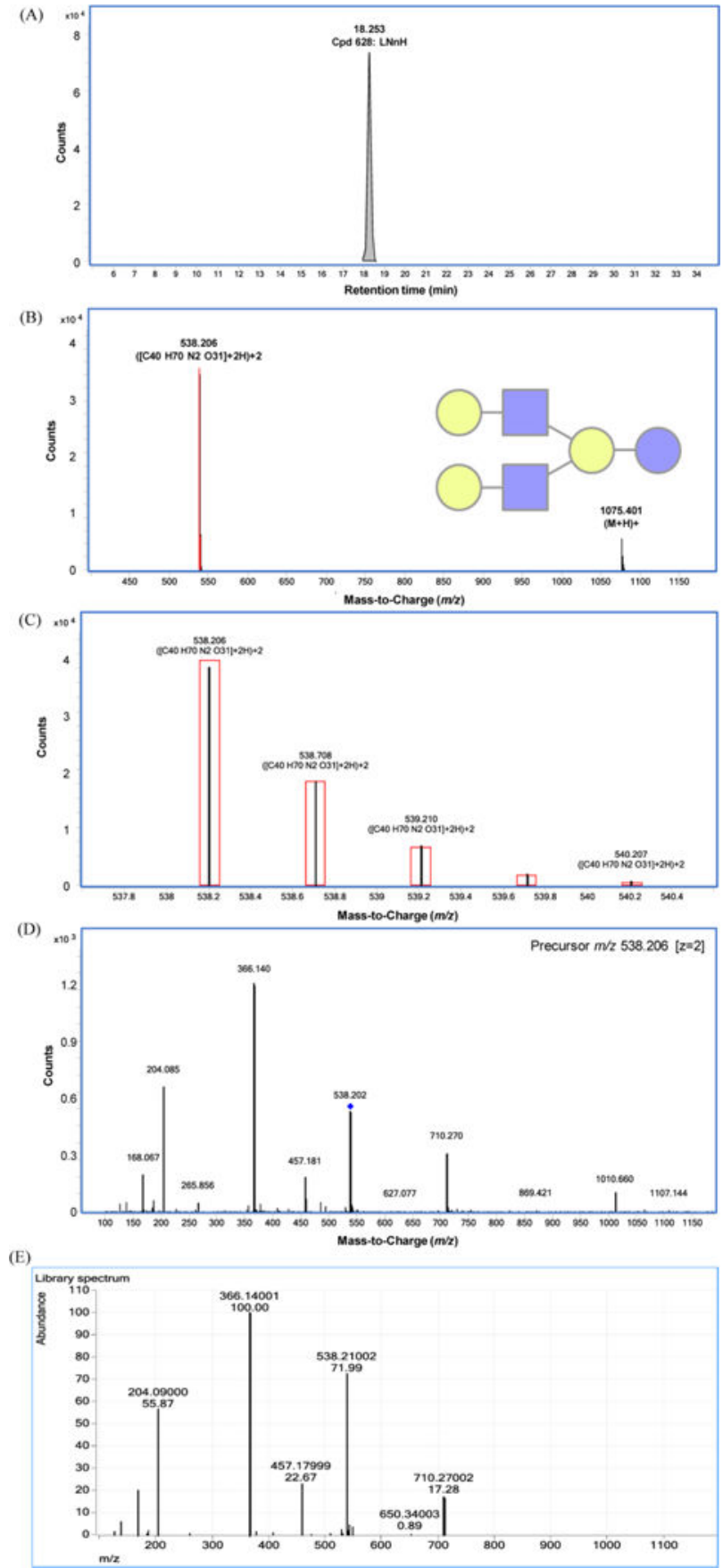

Figure 3.

Identification of $\mathrm{LNnH}$ in a whey permeate product using the custom PCDL. (A) Chromatogram identifying $\mathrm{LNnH}$; (B) an extracted mass spectrum for $\mathrm{LNnH}$ with the structures inserted; (C) zoomed-in image for the $\mathrm{LNnH}(\mathrm{m} / z$ 538.206) where the solid lines represent the observed mass spectra, and the overlaid boxes refer to the theoretical isotopic abundances; (D) MS/MS spectrum of a LNnH precursor ion ( $\mathrm{m} / z$ 538.206); and (E) an MS/MS spectrum from the library. 
(A)
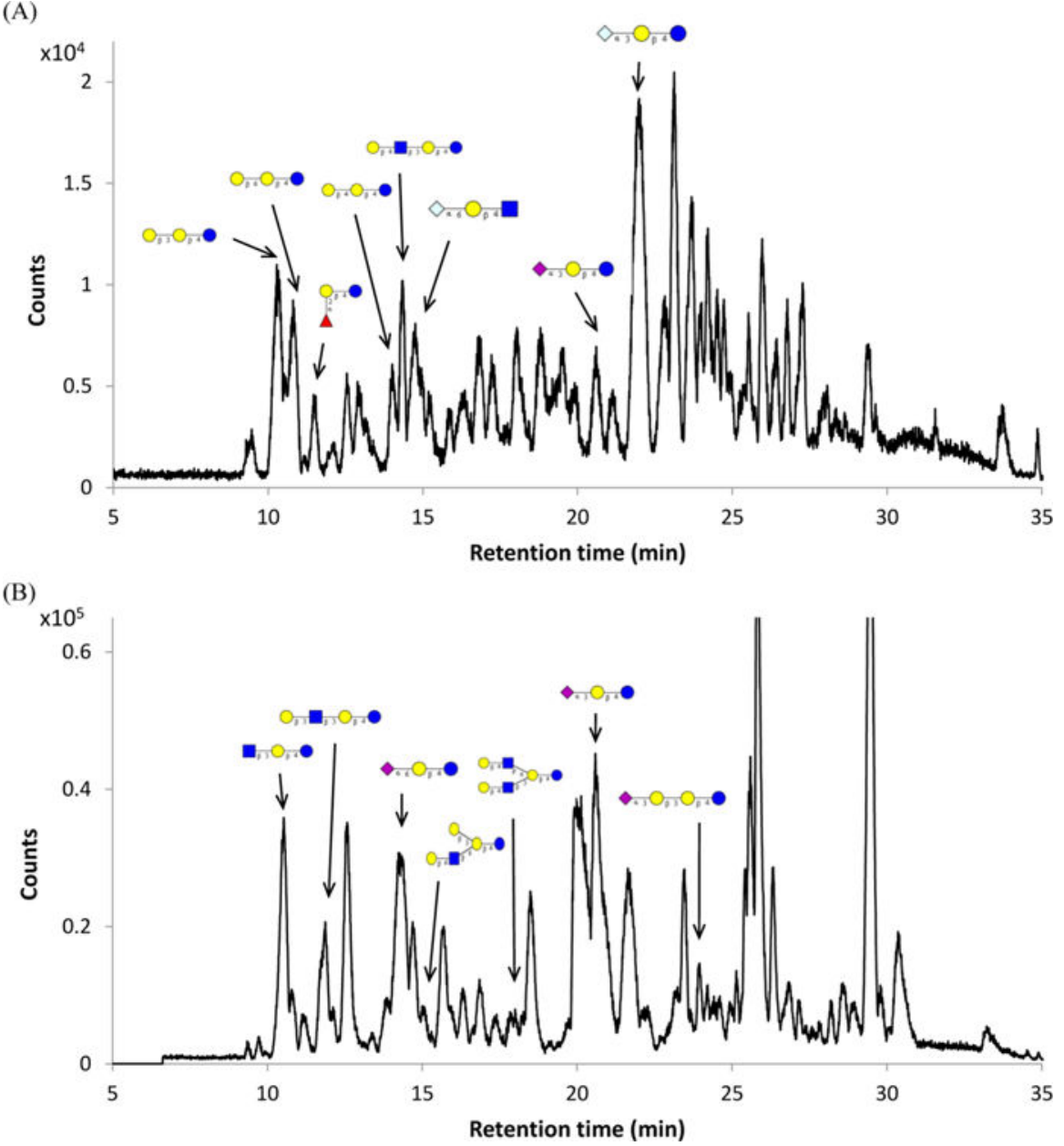

Figure 4.

Base peak chromatograms of oligosaccharides from (A) sheep milk and (B) buffalo milk with some structures annotated. 


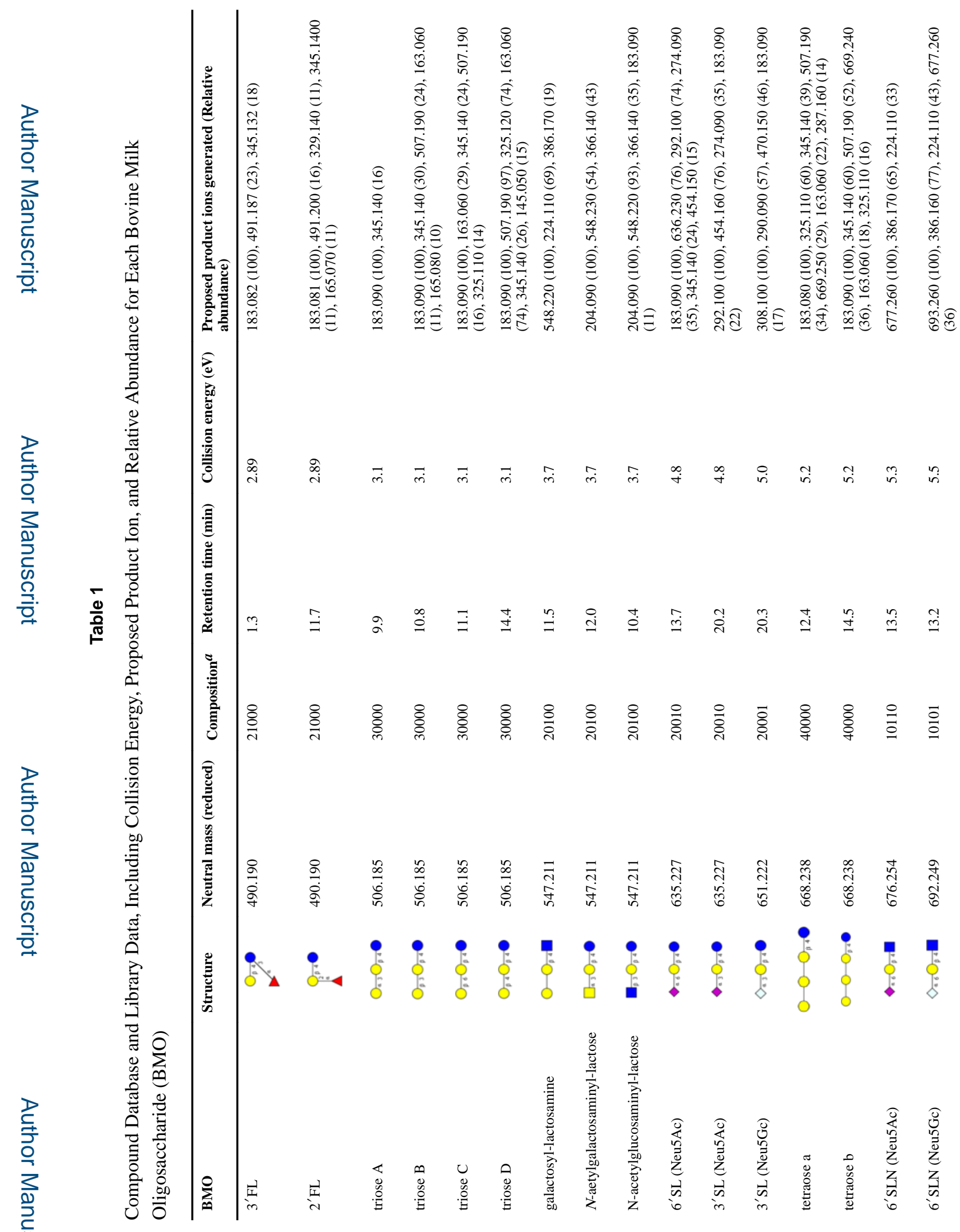




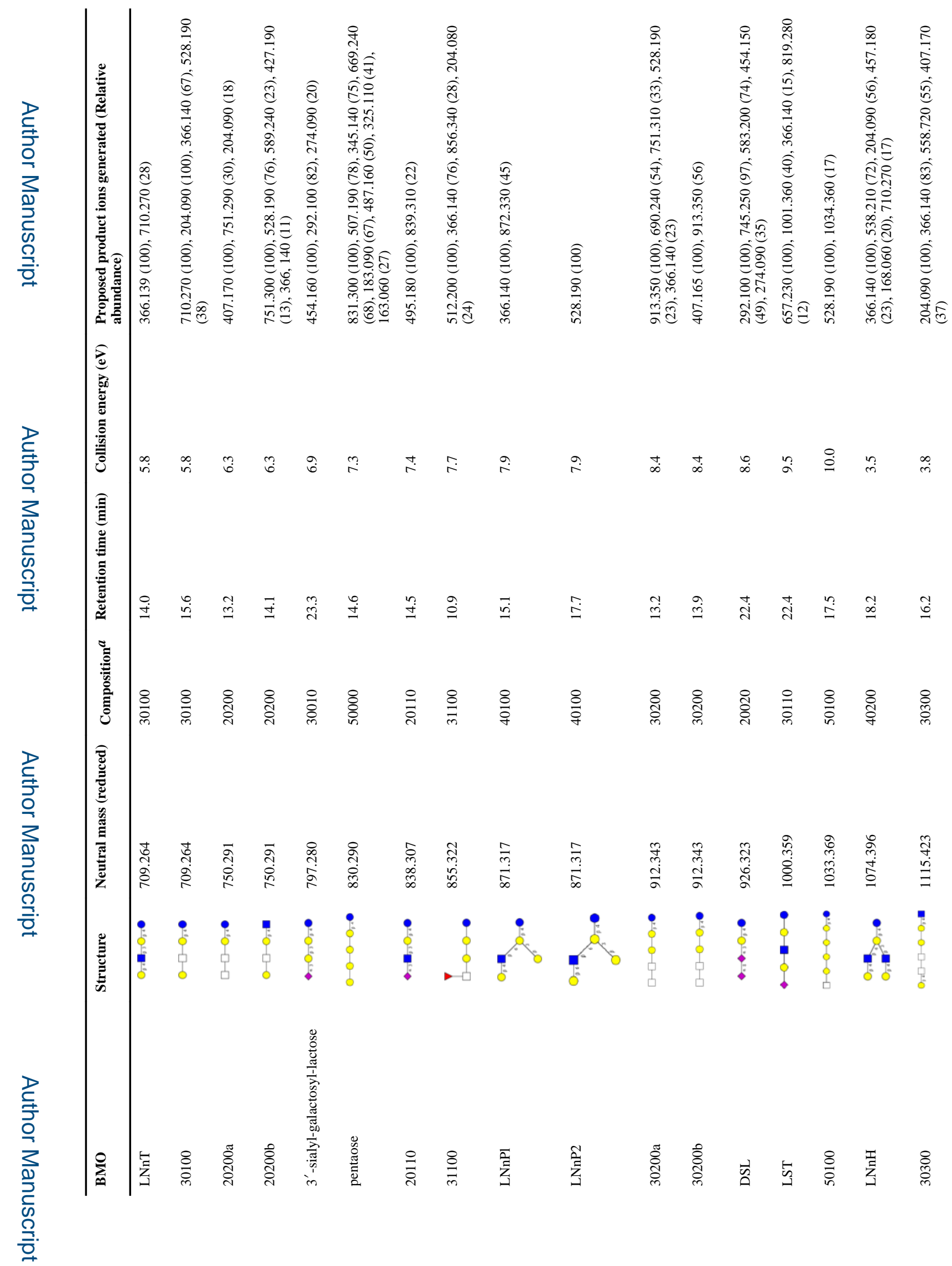

$J$ Agric Food Chem. Author manuscript; available in PMC 2018 March 01. 
Lee et al.

Page 18

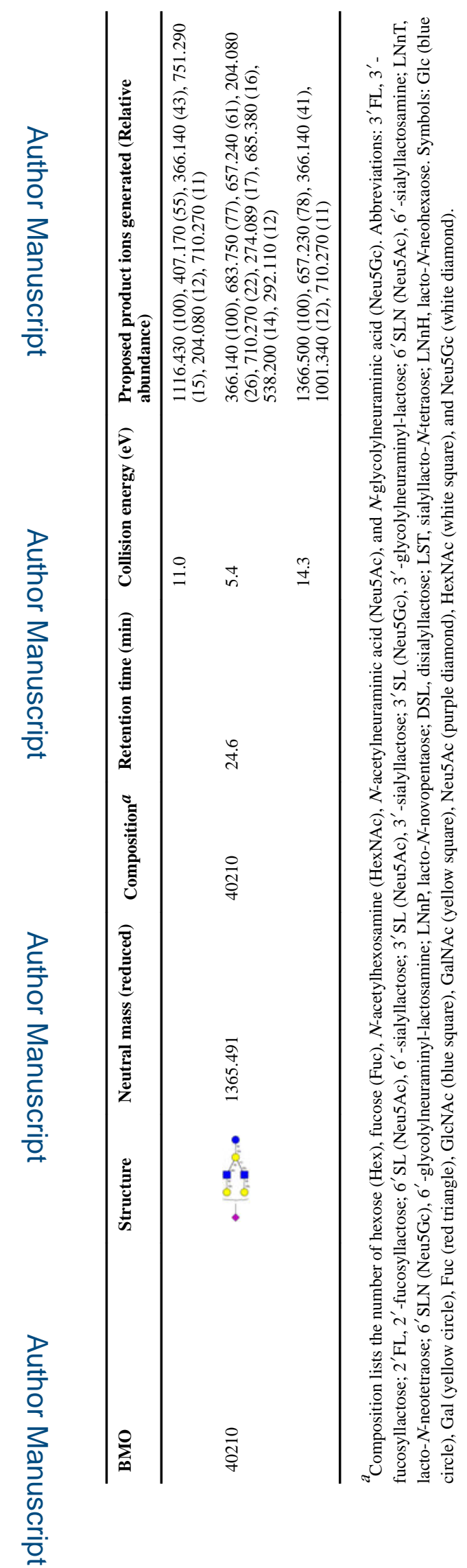

J Agric Food Chem. Author manuscript; available in PMC 2018 March 01. 


\section{Table 2}

(a) PCDL Search Results for $6^{\prime}$-Sialyllactose ( $\left.6^{\prime} \mathrm{SL}\right)$, Including the $\mathrm{m} / \mathrm{z}$ of the Precursor Ion and Matching Scores and (b) PCDL Search Results for $3^{\prime}$-Sialyllactose ( $3^{\prime} \mathrm{SL}$ ), Including the $m / z$ of the Precursor Ion and Matching Scores

\begin{tabular}{lcccc}
\hline & \multicolumn{5}{c}{ (a) } \\
name & precursor $\boldsymbol{m} / \boldsymbol{z}$ & collision energy (eV) & mass & score (\%) \\
\hline 6'SL (Neu5Ac) & 636.236 & 4.8 & 635.229 & 94 \\
3'SL (Neu5Ac) & 636.236 & 4.8 & 635.229 & 33 \\
\hline \multicolumn{5}{c}{ (b) } \\
\hline name & precursor $\mathbf{m} / \boldsymbol{z}$ & collision energy (eV) & mass & score (\%) \\
\hline 3'SL (Neu5Ac) & 636.236 & 4.8 & 635.229 & 98 \\
6'SL (Neu5Ac) & 636.236 & 4.8 & 635.229 & 35 \\
\hline
\end{tabular}

J Agric Food Chem. Author manuscript; available in PMC 2018 March 01. 


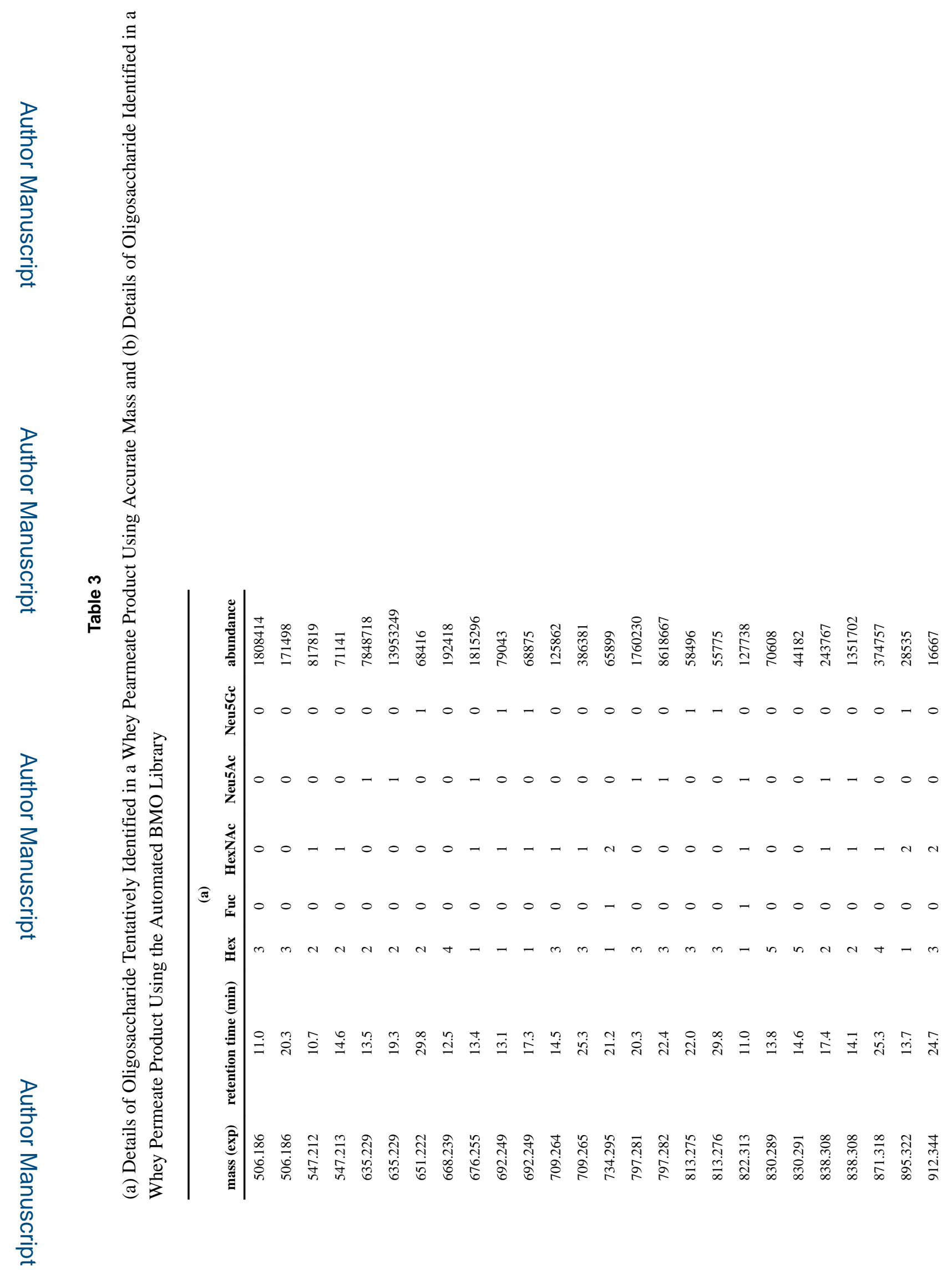

$J$ Agric Food Chem. Author manuscript; available in PMC 2018 March 01. 


\section{}

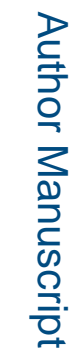

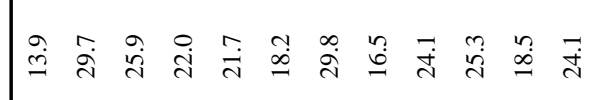




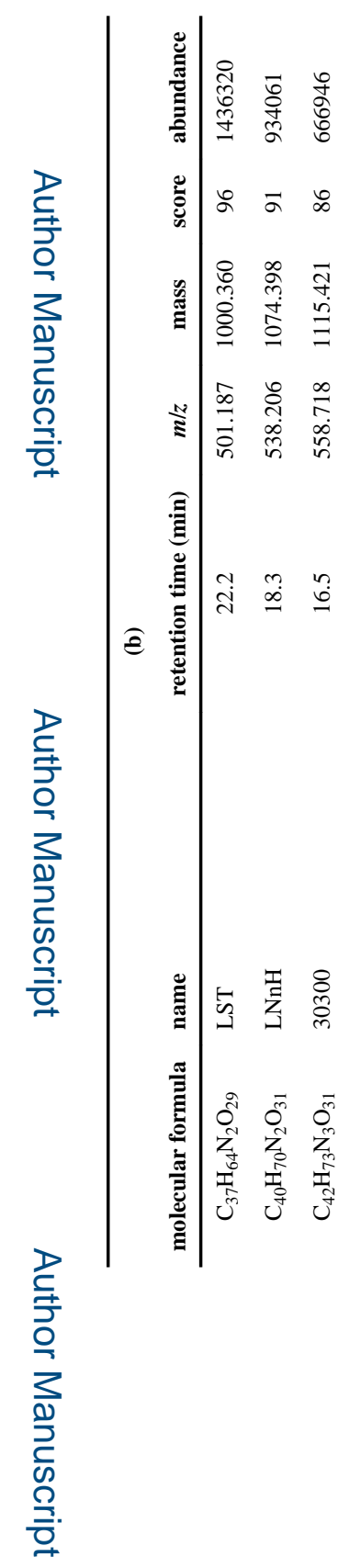

로을 